\section{Complejidades y desafíos sociales en iniciativas de conservación ¿Quién forma parte de la comunidad?}

Complexities and social challenges in conservation initiatives. Who is the local community?

\section{Daniela Serra-Maggi}

\section{Resumen}

Las Áreas Marinas Protegidas (AMP) son una importante estrategia frecuentemente usada para detener la sobre explotación de los recursos naturales. Uno de los desafíos con el que se encuentran las agencias e instituciones nacionales 0 internacionales encargadas de implementar áreas protegidas cuando quieren llevar a cabo procesos de participación, es identificar a los grupos de interés y dentro

Doctora en Antropología y Medio Ambiente Universidad de Otago, Nueva Zelandia. Tesis Doctoral Disponible en https://ourarchive. otago.ac.nz/handle/10523/3676 Correo electrónico: daniela. matakuri2@gmail.com de ellos a la comunidad local. Este artículo explorará en como grupos locales que en apariencia son homogéneos, muchas veces tienen estrategias económicas de sustento e identidades múltiples, complejas y dinámicas. Es por lo anterior que la falta de una adecuada incorporación de todos los actores locales claves, ha resultado en una afectación directa del bienestar de algunos grupos. Los grupos excluidos como participantes de la iniciativa de conservación, como son los del caso de este artículo, pierden derechos y acceso a recursos económicos necesarios para su sustento.

Palabras Clave: Antropología Ambiental, comunidad local, áreas marinas protegidas, dimensión social, participación

\section{Abstract}

Designated 'Marine Protected Areas' MPA are an important tool to stop the depletion of marine resources worldwide. A common challenge found by government and international agencies in charge of identifying MPA and deployment of them by the team who endeavors to carry out participation processes, is to identify the main stakeholders within the 'local community'. This paper explores how seemingly homogeneous local community groups actually have multiple, complex, and evolving identities, livelihood strategies, and sources. The inadequate attempt of MPA project staff to consider all the key local community groups has resulted in the exclusion of some groups. This situation has affected the well-being of the excluded groups and caused a failure to engage them as participants in the conservation initiative and it has imposed greater restrictions on access to coastal and 
marine resources upon which their livelihoods depend.

Key words: Environmental anthropology, local community, marine protected areas, social dimensions, participation

\section{Introducción}

Los problemas ambientales son inherentemente interdisciplinarios ya que el ambiente natural es afectado por factores sociales, culturales, químicos, físicos, políticos y económicos (Milton 1993). La Antropología Ambiental es una subárea de la Antropología Social y Cultural, la cual se concentra en el estudio de las dinámicas de interacción entre los humanos y sus ambientes naturales (Sievanen 2009; Carrier y West 2011). Esta sub-disciplina emerge en los 80 s, y a pesar que atrajo relativamente poca popularidad entre 1985 y el 2005, actualmente goza de altos niveles de interés (Kopnina and Shoreman-Ouimet 2013). La Antropología Ambiental juega un rol no solamente creando conocimiento relativo a sus áreas de estudio, sino también a través de su compromiso personal y defensa orientada a resolver problemas ambientales (Townsend 2000). Una de las características de esta defensa es la capacidad única de identificar los impactos sociales y culturales de los proyectos de conservación, por las ontologías específicas que los Antropólogos Ambientales hacen valer y las metodologías cualitativas que usan. Los proyectos bibliográficos de la Antropología Ambiental, tienen profundos e importantes impactos en el mundo alrededor de nosotros e inspiran investigaciones en terreno comprometidas y que promueven la justicia social y ambiental (Townsend 2008; West 2012; Lockyer y Veteto 2013; Shoreman-Ouimet y Kopnina 2016).
Los primeros estudios de la Antropología Ambiental se preocuparon de lo que el hombre hacía en su medio ambiente natural y como esto los afectaba a él, pero todavía estaba implícitamente entendido que el mundo se organizaba en dos reinos -el de la naturaleza y el de lo social- (por ejemplo: Evans-Pritchard 1940; Netting 1964, 1968 y 1972; Rappaport 1968). Aunque existen autores como Bateson (1979) que señalaron que esta distinción entre naturaleza y cultura no era tan productiva.

La 'nueva' Antropología Ambiental comienza con la deconstrucción de la separación de la sociedad en los dos 'reinos' binarios. En ella, estudios motivacionales fueron realizados sobre poblaciones locales involucradas en proyectos de conservación, los cuales buscaban modificar sus modos económicos de producción alterando sus modos de vida. En la última década del siglo veinte, e influenciados por la Antropología teórica postmoderna, los estudios antropológicos ambientales cambiaron a una visión que incorpora la motivación humana en su interacción con el medio ambiente natural como preocupación principal (Carrier y West 2011). Este cambio hacia los estudios motivacionales permitió a la Antropología Ambiental, como disciplina, destacar ambos - los impactos positivos y negativos - de las ideologías ambientales en las comunidades locales, en donde el objetivo principal es proteger el medio ambiente.

A continuación, se muestra un análisis de la literatura sobre como la exclusión de algunos grupos locales y la omisión de factores sociales y culturales, son una situación común que resulta de la idea de conservar el medio ambiente, a través de áreas protegidas. No obstante, estas áreas protegidas no consideran apropiadamente 
las dimensiones humanas, que es una situación negativa que ocurre muy a menudo. Distintas disciplinas de las ciencias sociales, entre ellas la Antropología Ambiental, pueden aportar para incorporar variables sociales y culturales que son importantes y se deben considerar en diversos proyectos de conservación ambiental para lograr implementarse adecuadamente.

\subsection{Establecimiento de áreas protegidas y residentes locales}

Las primeras ideologías del movimiento ambiental promocionaron la exclusión de las áreas de conservación de gente que usaba los recursos naturales de ellas a largo plazo y de manera permanente. Sobre todo, las áreas protegidas eran un mecanismo implementado como parte de un enfoque del movimiento ambientalista del hemisferio norte. Los científicos de las ciencias naturales-biólogicas, apoyaron las áreas protegidas como un instrumento adecuado y entregaron argumentos científicos a los gobiernos y a organizaciones internacionales para apoyar su desarrollo (Kenneth et al. 2008). Guerreiro et al. (2010) señala que las áreas protegidas eran vistas como instrumentos claves para resolver los problemas de destrucción de la biodiversidad y de extinción de especies alrededor del mundo. Varios momentos claves pueden ser identificados a nivel internacional que marcaron el momento en que empezó a promocionarse activamente la implementación de las áreas protegidas a nivel mundial, tal como la Conferencia Unida del Medio Ambiente Humano ${ }^{1}$ en 1972 y la Convención de la Diversidad Biológica (conocida por sus siglas en inglés CBD en 1992). Mientras algunas áreas protegidas fueron establecidas antes de

The United Conference on the Human Environment
1972, fue a través de la CBD que ellas fueron consolidadas como un plan mundial.

Sin embargo, a pesar de la exitosa promoción y apoyo internacional a las áreas protegidas, investigadores empezaron a identificar algunos efectos no beneficiosos de ellas en las comunidades locales (Guha 2000; Nepal 2002; Hutton et al., 2005; Wapner y Matthew 2009). Incluso antes que estos efectos negativos fueran evidenciados, respuestas a eventos de similares características, que incluían el prohibir los derechos de acceso a los recursos naturales alrededor del mundo de comunidades cuyos sustentos dependían de ellos, ilustraban la magnitud de los conflictos. Herrold-Menzies (2006) destaca como campesinos europeos históricamente se han opuesto a los deseos de la elite de apropiarse de sus tierras para proteger sus bosques y para cazar. En Asia, conflictos similares ocurrieron cuando los gobiernos coloniales erradicaron comunidades locales de los bosques para llevar a cabo la llamada 'ciencia forestal' (Agrawal y Gupta 2005; Agrawal 2005).

Residentes locales han sido igualmente excluidos de áreas protegidas en muchos países incluidos Estados Unidos E.E.U.U., India, y Australia. Guha (2000) explica la política de exclusión de comunidades locales de áreas protegidas en E.E.U.U. donde la protección de la naturaleza es una meta importante. La política de E.E.U.U. ha incluido erradicar al hombre y prohibir sus actividades productivas en estas áreas para la protección de parques y su fauna. La idea detrás de estas acciones es que el impacto humano va en contra de lograr la protección de la biodiversidad, por eso, el ingreso del hombre a estas áreas protegidas tiene que ser prohibido. Los resultados de la misma política en India ha implicado que cientos 
y miles de campesinos han sido desraizados de sus hogares, y miles más han dejado de tener acceso a recursos naturales restringiéndose sus fuentes de combustible, forraje y madera necesarias para su sustento (Guha, 2000). Ross et al. (2009) también explican que hasta las últimas décadas el establecimiento de áreas protegidas ha contribuido a la desposesión y marginalización de indígenas en Australia.

Sin embargo, la creación de áreas protegidas es una práctica estándar de los conservacionistas para lograr la protección de las áreas silvestres. De acuerdo a Wapner y Matthew (2009), la historia de las áreas protegidas está llena de muchos casos donde gente pobre fue erradicada para crear reservas y parques. Este es el caso, por ejemplo, de los indígenas norteamericanos en E.E.U.U., quienes fueron removidos para crear los parques Yosemite, Yellowstone, Gran Cañon, y Glaciar Nacional. Estas erradicaciones ocurrieron cuando ambientalistas, usualmente de los países desarrollados y con buenas intenciones, deseaban conservar la biodiversidad a través del establecimiento de parques y reservas sin tener en cuenta las necesidades de las poblaciones locales. A través de un caso de estudio de la Reserva Mexicana de la Biosfera Sierra de Huautla, Durand y Vázquez (2011) explican como proyectos de conservación han excluido a habitantes locales de su implementación. En este caso específico, en un análisis realizado por los autores se indica que autoridades de gobierno y científicos creían que para lograr conservar, la naturaleza debe ser mantenida con la menor intervención humana posible. Esta fue la justificación para que incluso las comunidades locales, a pesar de ser actores claves, fueran marginalizados de la administración no participando del diseño del proyecto de conservación.
Todos estos ejemplos ilustran situaciones de exclusión de recursos y hogares (Guha 2000, y Ross et al. 2009), donde argumentos sobre conservar la biodiversidad fueron utilizados para justificar los desplazamientos de los habitantes locales de las áreas protegidas (Wapner y Mathew 2009). Estos desplazamientos también conducen a una eventual exclusión de la gente local de los beneficios de los proyectos de conservación (Durand y Vázquez 2011).

Asimismo, el hecho de que el enfoque predominanteparaimplementaráreas protegidas afecta negativamente a gente indígena ha provocado reacciones en organizaciones indígenas. Una de estas situaciones ocurrió con La Coordinadora de las Organizaciones Indígenas de la Cuenca Amazónica (COICA), que llamaron a llevar a cabo acciones colaborativas entre las comunidades indígenas y los conservacionistas cuando su ecosistema amazónico era amenazado por el desarrollo y los proyectos de colonización que incluían crianza de ganado, tala ilegal de árboles, y operaciones mineras. COICA destacó que el movimiento conservacionista los había dejado afuera de la visión de la Biosfera Amazona, y esa exclusión impactó negativamente en ellos al no tener participación en la toma de decisiones de esas áreas protegidas. Es decir, sus intereses $y$ necesidades no fueron considerados $y$ esa es una de las razones principales por la que los programas establecidos por los conservacionistas han sido inefectivos (Chapin 2004).

La situación usual en la planificación de los proyectos de conservación es sólo incluir información biológica, con a veces ocasionalmente información económica. Sin embargo, hay una falta permanente de 
información social y cultural, porque obtenerla requiere investigaciones que a menudo toman mucho mayor tiempo de conseguir que la información biológica o ecológica. No obstante, existe una amplia literatura criticando los proyectos de conservación la cual establece que la inclusión de las poblaciones locales y de sus factores sociales y culturales en el desarrollo y manejo de proyectos de conservación para el manejo de los recursos naturales es primordial (por nombrar algunos: Guha 2000; Nepal 2002; Chapin 2004; West 2006; Ross et al. 2009; Schmidt y Paterson 2009; Wapner y Matthew 2009; Durand y Vázquez 2011; Bennett et al. 2016). No obstante cabe destacar, que hoy en día en el establecimiento de áreas de conservación de la naturaleza, existe una diversidad de categorías/tipologías de implementación. Estas tipologías van desde áreas protegidas donde no se permiten los múltiples usos, ni la participación en ninguna forma de las comunidades locales y sólo se lleva a cabo una estricta conservación, hasta otras donde la participación local y el desenvolvimiento económico de la comunidad son los aspectos fundamentales de la estrategia de conservación. En el mundo y a lo largo de la historia, la mayor cantidad de áreas de protección y conservación implementadas se han basado en el tipo de conservación estricta, sin participación comunitaria, pero hoy en día la mayoría de los nuevos proyectos de conservación buscan algo más cercano al otro extremo de las tipologías señaladas, incluso incluyendo el co-manejo o co-administración en conjunto con las comunidades para la administración de las áreas protegidas.

Mientras ha habido un avance sustantivo en el involucramiento de las comunidades en los proyectos de conservación, no ha avanzado de la misma forma el incluir las dimensiones sociales en los proyectos de conservación. A pesar de que la literatura ha enfatizado la necesidad de reconocer el rol de los factores sociales y culturales, y las políticas de las agencias de financiación han reforzado este reconocimiento, esto no siempre se ha hecho evidente en la práctica. Por eso, otra situación que ha sucedido comúnmente es el que los encargados de implementar los proyectos de conservación, no comprenden la complejidad de las situaciones y realidades sociales y socioeconómicas e incluso políticas existentes donde se implementan los proyectos. Un ejemplo de esto es el de la Antropóloga norteamericana West (2006), quien investigó sobre los programas de conservación implementados por Organismos No Gubernamentales (ONGs) en Goroka, Papua Nueva Guinea, advirtiendo como las iniciativas de conservación impactaban a las comunidades locales por la forma en que estas entendían el medio ambiente y la sociedad. En este caso específico, los locales tenían la expectativa de que los ejecutores del proyecto de conservación les proveyeran deservicios que debía entregarles el gobierno, relacionados con educación, salud, y desarrollo socio-económico, entre otros. El rol de gobierno de las ONGs no fue logrado por ellas, pues no era su objetivo, causando que las comunidades se decepcionaran mucho con los administradores del proyecto de conservación. Los que diseñaron el proyecto estaban principalmente preocupados de la conservación de la biodiversidad biológica, y el proyecto fue planificado usando un enfoque de arriba hacia abajo, lo que implica que no tenía inputs de la comunidad local. En este caso, el uso de información socio-económica y cultural habría entregado información clave para haber podido mitigar las altas expectativas de la población local. Por otro lado, un ejemplo latinoamericano sobre el rol de las ONGs internacionales en 
la conservación se encuentra en Diegues et al. 2008 donde los autores describen como las ONGs a través de una agenda de conservación predominantemente basada en lo ecológico lograron proteger la biodiversidad de varios territorios, pero a costa de descuidar la participación de las comunidades locales y de no considerar aspectos sociales de ellas como parte de las soluciones a problemáticas ambientales que buscaban subsanar.

\subsection{Establecimiento de Áreas Marinas Protegidas}

Dentro de las áreas protegidas también se encuentran las Áreas Marinas Protegidas (AMP), estas también son una importante herramienta de conservación para detener la sobre explotación de los recursos marinos a nivel mundial y para conservar la biodiversidad (Kelleher y Recchia 1998) Sanchirico et al. 2002; Banks y Skilleter 2010; Ma et al. 2013). En los últimos 20 años las AMP han proliferado alrededor del mundo y actualmente según una lista de Las Naciones Unidas cubren un $3.41 \%$ de las áreas marinas en el mundo (Deguignet et al. 2014). Numerosas investigaciones han demostrado que las AMP entregan beneficios para la conservación de la biodiversidad (Halpern et al. 2008). Sin embargo, también se ha encontrado que muchas de estas áreas protegidas causan efectos negativos en comunidades locales de pescadores artesanales, generalmente porque no consideran adecuadamente en su implementación factores sociales y culturales de ellos (Cinner et al. 2010; Bennett y Dearden 2014). Por esta situación, han sido adoptados nuevos enfoques para la implementación de AMP, estos incluyen la participación de comunidades locales e investigaciones sobre las dimensiones humanas de ellas. Gran parte del éxito de la implementación de las AMP depende de su legitimidad entre las poblaciones locales que hacen uso de los recursos marinos, por eso hay que lograr la cooperación local en la conservación de estos recursos (Pinkerton y John 2008). En este sentido, estudios han señalado que la planificación de las AMP tiene que ser basada considerando las dinámicas de poder y el bienestar de las comunidades que dependen de los recursos marinos que se quiere proteger con la creación de estas áreas (Gelcich et al. 2005) Mascia et al. 2010; Cinner et al. 2010).

Las AMP incluyen una gama de tipologías que fluctúan desde la exclusión de cualquier usuario del uso y manejo de ellas, hasta el acceso y uso de los recursos marinos entre individuos y grupos y manejadas integralmente por usuarios locales. Lo más común ha sido la proliferación de áreas marinas más cercanas a la exclusión de usuarios. No obstante, hoy en día se está defendiendo y buscando implementar las que si permiten usos, debido a que esta tipología busca asegurar que se tenga una participación efectiva y la representación de todos los grupos claves para la entrega de acceso y beneficios. Esta tipología de área marina protegida, cree que de esta forma se reduce la posibilidad de crear conflictos y evitar cambiar el equilibrio que hay entre todos los que hacen uso de los recursos marinos.

Analizando lo que dice la literatura al respecto, tenemos a Mascia et al. (2010) que discute, en relación a las AMP con su contexto local, que las decisiones políticas deberían estar basadas en investigaciones sobre dimensiones humanas como el poder político y el bienestar social de las comunidades de pescadores. Según el autor, a pesar de que las AMP podrían ser una buena opción para mejorar y empoderar a las 
comunidades locales y asegurar su sustento, éstas han probado tener efectos perjudiciales para las comunidades de pescadores. Por otro lado, Brooks (2010) enfatiza que la literatura sobre políticas marinas y manejo de recursos marinos se beneficiaria de la inclusión de los "elementos sociales" en la evaluación de iniciativas de conservación.

A través de la revisión de la literatura sobre manejo de ecosistemas marinos Curtin y Prellezo (2010), también demuestran como los objetivos ambientales y económicos son considerados y logrados mientras los aspectos sociales se mantienen sin resolver. Los humanos son parte del ecosistema marino y costero, lo que indudablemente implica que pueden afectar y transformar esos sistemas con sus acciones. Brooks (2010) también provee de un ejemplo de la importancia de los aspectos "sociales" a través de una investigación llevada a cabo en la Pesquería Marina de Australia del Sur. Aquí destaca como resultado que para lograr el desarrollo sustentable, es necesario tener éxito en tres áreas que potencialmente se sobreponen: la económica, ambiental y social. En esta investigación la social fue la menos lograda.

El caso de estudio que se presenta en este artículo es uno de los ejemplos que existen, que demuestra que la exclusión de algunos grupos locales en un plan de conservación que consiste en crear un tipo de AMP, es una respuesta común a la idea de comunidades que viven y mantienen su sustento económico de estas áreas. Por eso conocer quién forma parte de la comunidad o grupos locales se hace indispensable. Esta pregunta es parte de un debate amplio que gráfica la tensión entre conservación-cultura en la implementación de áreas protegidas. Más aún, existen muchos autores que señalan que la identificación de los grupos locales para el involucramiento y participación de ellos en las áreas protegidas, es crítico para el éxito de la implementación y su objetivo de conservar los recursos naturales (Blomley 2015; Koehn et al. 2013; Berkes 2004; Agrawal 1999; Brosius et al 1998). En el caso de este artículo, se hacía vital para no haber excluido a ningún grupo importante, conocer 'quién era la comunidad' y en eso la Antropología como disciplina podría haber aportado de manera significativa como lo veremos en las próximas secciones.

\section{Caso de estudio chileno de Área Marina Protegida}

Para este artículo se analiza un caso chileno de creación de Áreas Marinas Costeras Protegidas de Múltiples Usos (MU) creadas en Chile entre el 2005 y el 2009. Las Áreas Marinas Costeras Protegidas-MU son un proyecto financiado por el Fondo Global Ambiental (GEF) basado en la Estrategia Nacional de Protección de la Biodiversidad y son parte de la red de áreas marinas protegidas que el gobierno de Lagos planificó crear. Previamente, la legislación para la protección del mar y la costa en Chile estaba separada. Por ende, el gobierno de Chile creó una nueva regulación especial para el establecimiento de las áreas que protegieran simultáneamente el mar y la costa. Las Áreas Marinas Costeras Protegidas-MU están destinadas a albergar diversas actividades, tales como: eco-turismo, pesca artesanal y áreas de conservación.

El GEF administró las Áreas Marinas Costeras Protegidas-MU junto con el Programa de Naciones Unidas para el Desarrollo (PNUD) y el Gobierno de Chile en un Programa llamado "Conservando Globalmente Biodiversidad 
Prioritaria a lo largo de la Costa"2 también conocido como el proyecto GEF Marino. En el 2005, el Gobierno de Chile creó tres Áreas Marinas Costeras Protegidas-MU en diferentes lugares de Chile en las regiones de Atacama, Los Lagos y de Aysén (Figura 1).

Figura 1. Mapa de Chile y ubicación de las tres Áreas Marina Protegidas-MU creadas.

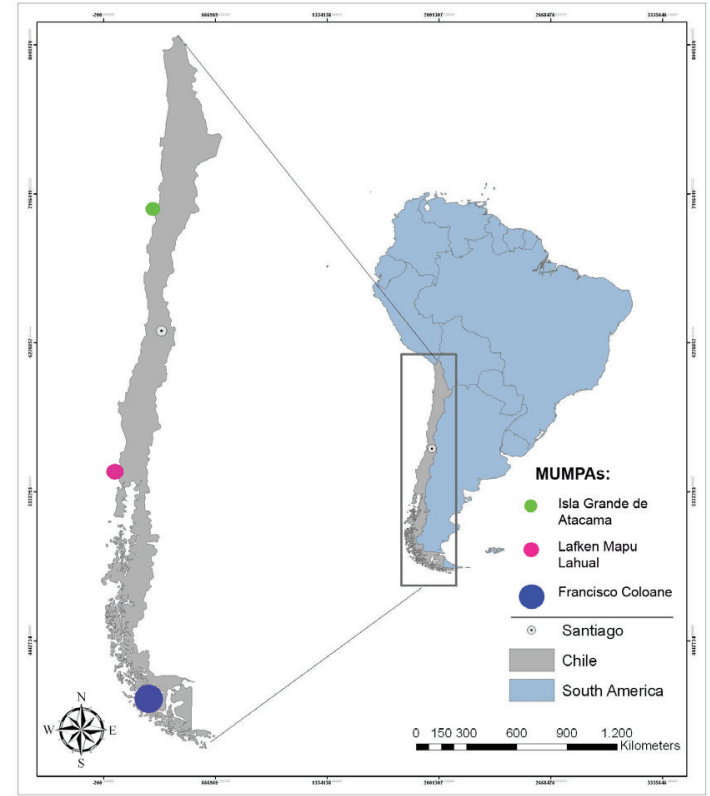

Fuente: elaboración propia.

El objetivo principal de las Áreas Marinas Costeras Protegidas-MU es proteger y conservar la biodiversidad. Una de las estrategias llevadas a cabo para lograr la tarea de conservar fue desarrollar estrategias socioeconómicas que incluían un proyecto piloto

"Conserving Globally Significant Biodiversity along the Chilean Coast" de eco-turismo dirigido a las comunidades locales que dependían económicamente de la biodiversidad costera y marina que se pretendía conservar. Se pensó que el ecoturismo generaría ganancias que ayudarían a monitorear estás áreas protegidas. Por ende, las Áreas Marinas Costeras Protegidas-MU iban a proteger los recursos marinos costeros restringiendo el acceso a éstas áreas a toda actividad que no fuera de pesca sustentable y eco-turismo. Esta actividad iba a también a contribuir a disminuir la pobreza de la gente local creando alternativas que implicaban usar los recursos naturales sin consumirlos a través del eco-turismo. Parte de la infraestructura prometida por el GEF Marino para el desarrollo del turismo del área protegida creada, por ejemplo, para el área de la Región de Los Lagos consistía en: embarcadero, helipuerto, centro de visitantes, casa de administración, casas de guarda parques, garitas de acceso al área marina, cabañas de turismo, agua potable, camino y senderos turísticos.

En la región de Los Lagos el área creada se llamó Lafken Mapu Lahual y de las tres Áreas Marinas Costeras Protegidas-MU creadas en Chile, esta fue la única donde hubo participación de comunidades indígenas. El área Lafken Mapu Lahual está ubicada en la Comuna de Río Negro, Provincia de Osorno (Figura 2), frente al bosque templado que se encuentra a lo largo de la cordillera de la costa en un área conocida como Territorio Mapu Lahual. La World Wide Fund for Nature (WWF) ha designado ésta área de bosques lluviosos como un área prioritaria de conservación ya que está caracterizada por una enorme biodiversidad, incluyendo especies endémicas en peligro de extinción de flora y fauna (WWF, 2007). El área total protegida Lafken Mapu Lahual es de 5,431 hectáreas, 
incluyendo aproximadamente 4,463 hectáreas de mar. Tiene 32 kilómetros de largo y tiene una milla de ancho. Esta área marina y costera incluye los ríos Huellelhue y Cholguaco.

Para esta investigación los dos principales grupos estudiados son: (i) los indígenas especializados en el alerce, que se están convirtiendo en pescadores como una segunda ocupación complementaría a la del trabajo del alerce, y; (ii) los pescadores históricos, que pueden ser indígenas o no, pero que no son miembros de ninguna comunidad indígena.

Dentro del primer grupo son consideradas nueve comunidades indígenas del Territorio Mapu Lahual. (Figura 2). La tala del alerce muerto es la fuente principal de ingresos para la mayoría de estas comunidades. En el idioma MapucheHuilliche, "Territorio Mapu Lahual" significa "Mapu" = Tierra y "Lahual" = Alerce, por lo cual el nombre de este territorio es "Tierra del Alerce.

Figura 2. Ubicación de las nueve comunidades indígenas del Área Marina Protegida Lafken Mapu Lahual.

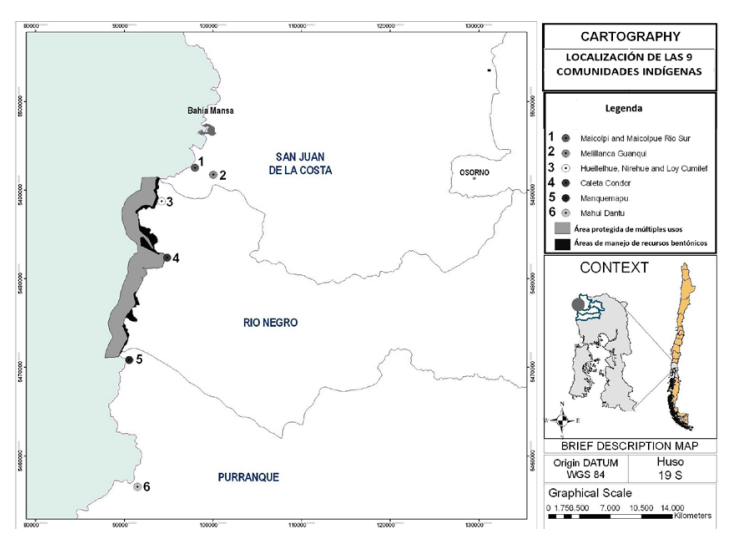

Fuente: Elaboración propia.
En este primer grupo fueron entrevistados los líderes de las nueve comunidades indígenas del Territorio Mapu Lahual. El propósito de la recolección de estas narrativas fue recopilar el discurso de las cuatro comunidades indígenas que fueron los actores principales en la implementación del Área Marina Protegida Lafken Mapu Lahual: Caleta Cóndor, Caleta Huellelhue, Nirehue y Loy Cumilef. Sin embargo, también dentro de este grupo son consideradas las otras cinco comunidades indígenas que también son parte del Territorio Mapu Lahual, pero que a pesar de no recibir beneficios para sus comunidades si participaron de la implementación del área protegida a través de la Asociación Indígena Mapu Lahual y de la Red de Parques Indígenas Mapu Lahual.

En el segundo grupo son consideradas dos meta-organizaciones de pescadores que se señalan en la siguiente Tabla 1:

Tabla 1: meta organizaciones de pescadores

\begin{tabular}{|l|}
\hline \multicolumn{1}{|c|}{ Asociaciones de pescadores artesanales } \\
\hline \multicolumn{1}{|c|}{ Meta organización de pescadores: CPALO } \\
\hline Coopemar Trumao CooCooperativa Coopemar Trumao \\
\hline Sindicato N² \\
\hline Sindicato Maicolpué Río Sur \\
\hline $\begin{array}{l}\text { Asociaciones de pescadores de Huellelhue, Nirehue y } \\
\text { Loy Cumilef }\end{array}$ \\
\hline Asociación de pescadores Caleta Cóndor \\
\hline Sindicato Playa Centro \\
\hline \multicolumn{1}{|c|}{ Meta organización de pescadores: FEDEPESCA } \\
\hline Sindicato N 1 \\
\hline Sindicato N ${ }^{\circ} 4$ \\
\hline Sindicato Encarnadoras \\
\hline Sindicato Pucatrihue \\
\hline Sindicato Costa Brava \\
\hline
\end{tabular}

Fuente: elaboración propia. 
Dentro de este segundo grupo fueron entrevistados los líderes de las organizaciones de pescadores. El propósito de la recolección de las narrativas de estos pescadores fue el recopilar el discurso del segundo grupo de actores más importante para la implementación de la Área Marina Protegida-MU. Una parte de este segundo grupo, los miembros del Consejo de Pescadores del Litoral de Osorno (CPALO), no fueron los participantes principales del área marina protegida, pero si fueron involucrados en la iniciativa y por ende recibieron algunos beneficios. Otros miembros de este segundo grupo, la Federación de Pescadores (FEDEPESCA), fueron excluidos del área marina protegida.

El nivel de compromiso y participación local de los indígenas y pescadores en la protección del Área Marinas Costera Protegida-MU fue evaluada a través de una metodología etnográfica, durante siete meses de terreno, donde entre otras técnicas de investigación fueron llevadas a cabo 37 entrevistas individuales semi-estructuradas. El análisis de estas entrevistas mostró la existencia de diferentes discursos y la posición de estos discursos en conflicto, los cuales se enmarcan y construyen dentro de una realidad y contexto particular durante la implementación de la Área Marina Costera Protegida-MU Lafken Mapu Lahual.

\section{Resultados}

\section{Implicancias socio-culturales del Área Marina Protegida-MU Lafken Mapu Lahual}

Esta investigación ha identificado un set específico de aspectos socio-culturales que no han recibido suficiente atención en las iniciativas que integran objetivos de conservación y desarrollo desde que empezaron a establecerse (Hughes y Flintan 2001; Chapin 2004), como ha ocurrido con el Proyecto GEF Marino que creó el Área Marina Costera Protegida-MU Lafken Mapu Lahual en nuestro país desde el 2005.

A continuación, se explican tres de esos aspectos que influyeron en aumentar conflictos entre pescadores artesanales y comunidades indígenas y que causaron el no contar con los elementos esenciales y estrategias para poder promover una buena participación y compromiso de las comunidades locales para lograr el éxito del Área Marina Protegida-MU Lafken Mapu Lahual.

a) Trabajar exclusivamente con dos organizaciones locales que el staff del proyecto GEF Marino erróneamente determinó que representaban a la mayoría de los principales actores locales.

b) Presunciones incorrectas sobre los antecedentes culturales de las comunidades indígenas.

c) Presunciones incorrectas sobre el compromiso de lascomunidades indígenas con actividades de conservación.

A continuación, se explican estos aspectos sociales detectados durante la investigación:

\section{a) Trabajar exclusivamente con dos organizaciones locales que el staff erróneamente pensó que representaban a la mayoría de los principales actores locales.}

La falta de una real participación de todas las comunidades locales impactó la implementación del Área Marina Costera Protegida-MU Lafken Mapu Lahual. Iniciales niveles de participación, 
por ejemplo, haber sido informado sobre el proyecto, eran importantes primeros pasos para legitimar el proyecto. Sin embargo, algunos grupos como FEDEPESCA expresaron que no fueron consultados o involucrados y ni siquiera considerados como un grupo local importante. Cuando ellos eran los pescadores más históricos y tenían tantas necesidades económicas como las comunidades indígenas y, más aún, su sustento dependía de los recursos marinos que se encontraban en el área marina protegida. Por lo tanto, de acuerdo a las comunidades locales, el grado de participación del GEF Marino fue insuficiente. En contraste, el staff del proyecto explicó en sus entrevistas que si había habido una adecuada participación. Estas diferencias de opinión son significativas, considerando que la participación en cualquier tipo de proyecto de conservación es considerada como un aspecto crucial y determinante del éxito.

\section{b) Presunciones incorrectas sobre los antecedentes culturales de las comunidades indígenas.}

Las comunidades indígenas de Caleta Cóndor, Huellelhue, Nirehue y Loy Cumilef fueron elegidas por el staff del proyecto como el principal actor local y el que iba a estar encargado de administrar el área marina protegida, lo que significaba que la mayoría de los beneficios, aunque no todos, fueran para ellas. No obstante, hubo características de las comunidades indígenas elegidas como actor principal que afectaron el éxito de la implementación del área protegida Lafken Mapu Lahual que incluyen: (i) Los miembros de las comunidades indígenas no han residido suficiente tiempo en las comunidades como para haber desarrollado un conocimiento tradicional ecológico sobre la costa y el mar, por ende, ni su identidad ni sustento se basa en el mar; (ii) No tenían una identidad cultural indígena expresada en su idioma y sus costumbres sino más bien rural-forestal; (iii) La mayoría, no son residentes de las comunidades indígenas, pues viven en Bahía Mansa, no en sus comunidades; (iv) No tienen características culturales diferentes a la gente local no indígena de la zona, ya que los miembros provienen de distintas partes de la región; y (v) Su conocimiento tradicional ecológico está relacionado con el alerce e incluye prácticas que algunas veces no son sustentables. Ninguno de estos factores sociales y culturales fue considerado desde un punto de vista antropológico, a pesar de lo crucial que podrían haber sido estos elementos para la implementación y éxito del Área Marina Costera Protegida-MU. Estas características obstaculizaron el compromiso y la participación de las comunidades indígenas. Claramente su consideración era fundamental para el éxito en la implementación de un área marina protegida.

\section{c) Presunciones incorrectas sobre el compromiso de las comunidades con actividades de conservación.}

Por otro lado, el área marina protegida estaba basada en supuestos sociales y culturales que se identificaron a través de las entrevistas realizadas durante la investigación, las que resultaron en una falta de coherencia en la implementación del proyecto. Estos supuestos incluían que las comunidades indígenas que eran los participantes principales del proyecto estaban comprometidos y participando previamente en conservación con su involucramiento activo en la Red de Parques Mapu Lahual y que estas comunidades indígenas todavía mantenían sus costumbres y características culturales indígenas. El staff del AMP no consideró estas características, porque no contaba con una línea 
base social y cultural antes de implementar el proyecto, aunque contaron con una a posteriori, cuando ya el proyecto estaba en proceso de implementación, lo que pudo haberles entregado los conocimientos sobre estas comunidades indígenas desde un principio. De la misma forma, al Área Marina Protegida-MU le faltó considerar las necesidades socio-económicas de éstas comunidades. El no tener en cuenta estas características culturales, y tener estos supuestos incorrectos, la falta de entendimiento de las necesidades de sustento, y el incumplimiento de las promesas (de la infraestructura eco-turística) y expectativas se combinaron causando la falta de deseo de los pescadores artesanales y de las comunidades indígenas de comprometerse y participar para poder administrar y cuidar el área marina protegida. Por otro lado, la prioridad de las comunidades indígenas elegidas para administrar el área protegida no era participar en proyectos de conservación. Uno de los principales problemas de los miembros de las comunidades indígenas era que no eran dueños de sus tierras en las comunidades indígenas, por lo tanto, tenían necesidades y problemas más urgentes que resolver que estar participando de la implementación y administración del Área Marina Costera Protegida-MU Lafken Mapu Lahual.

Esta investigación muestra como en la implementación de Área Marina Costera Protegida-MU Lafken Mapu Lahual, se dieron todas las situaciones mencionadas en la introducción: falta de compromiso en la protección y administración del área protegida creada por las comunidades y grupos locales; afectación directa del bienestar, en este caso de un grupo de pescadores FEDEPESCA 'los históricos', al prohibírsele los derechos de acceso a los recursos marinos del área marina protegida creada, por ende, el ser excluidos de la implementación, beneficios, acceso y uso del área; no tenerse en cuenta las necesidades de todos los principales grupos locales, privilegiando a unos por sobre otros lo que generó resentimiento, frustración y conflicto entre ellos; hacerse un proyecto desde arriba hacia abajo, lo que implica no tener inputs de las comunidades locales en el diseño del proyecto; ausencia de legitimidad de la creación del área protegida por parte de los principales grupos locales; falta de mejoramiento del empoderamiento de las comunidades locales y aseguramiento de su sustento; y creación de altas expectativas de que a través del área marina protegida se podía mejorar el nivel de ingresos, y por ende, la situación económica de las comunidades locales, proveyéndolas de mejoras en infraestructura que deberían haber sido aportadas por el gobierno y no por un proyecto de conservación.

Toda esta situación ocurrió porque las identidades de los actores eran múltiples, complejas y dinámicas, lo que convertía en un gran desafío para el staff del proyecto de conservación el conocer a cabalidad el contexto, realidad social y cultural respecto al lugar y sus habitantes.

\section{Reflexiones y consideraciones finales}

Respondiendo a la pregunta inicial planteada en este artículo ¿Quién forma parte de la comunidad o grupos locales?" Los resultados de esta investigación muestran un caso particular, en un contexto específico como es el Área Marina Costera Protegida-MU Lafken Mapu Lahual, focalizando su atención en características culturales y sociales, así como 
en las dinámicas de las comunidades locales que potencialmente eran los actores más relevantes en la implementación del AMP. Para responder esta pregunta, los implementadores debían haber conocido a fondo todos estos factores locales. Es el análisis exhaustivo de estos elementos, lo que hubiera hecho comprender las características del grupo que iba a formar parte de la implementación del AMP y las implicancias de dejar a otros grupos de la comunidad de lado o en segundo lugar durante el establecimiento del área.

Estas características y dinámicas no han sido consideradas extensamente en la literatura científica, salvo casos como Chuenpagdee et al. (2013). Estos autores señalan a través de cuatro casos de estudio de México y España, situaciones como las de nuestro artículo en donde en la fase inicial (llamada paso zero), previa a la implementación del AMP, en donde la idea es concebida, comunicada y discutida, existen riesgos políticos y conflictos entre los distintos grupos de interés.

De forma de evitar estas situaciones descritas en este artículo, actualmente encontramos enfoques que promueven una fuerte innovación en la gobernanza y protección de los océanos como herramientas de diagnóstico transdisciplinarias, donde, entre otras iniciativas y acciones, se reconozca a los seres humanos como una parte integral de las complejidades socio-ecológicas y sus soluciones, se considere una mirada de abajo hacia arriba, con mucho input de las comunidades en la entrega de conocimiento y gestión en el ámbito marino (Gerhardinger et al. 2018).

En nuestro caso de estudio, a pesar de las grandes capacidades técnicas y buenas intenciones del staff implementador del Área Marina Costera Protegida-MU Lafken Mapu Lahual, por ser estas sólo en el área biológica y de pesca, los riesgos políticos y los conflictos locales no fueron conocidos o considerados por los implementadores y por ende menos gestionados. En consecuencia, elementos políticos, conflictos y dinámicas sociales locales tienen el potencial de influenciar de gran manera la implementación y el éxito de una iniciativa de este tipo. Estos factores, en esta investigación, incluyen conflictos de tierras, etnicidad, situación económica de grupos locales, nivel de educación, conflictos entre pescadores y comunidades indígenas, contexto político local y conflictos entre organizaciones locales. Aunque para este artículo sólo se describió algunas de estas situaciones. Los resultados de esta investigación muestran que uno de los principales problemas con el Área Marina Costera Protegida-MU fue la inclusión y consideración de estos factores sociales, aunque no el único.

Esta investigación ha resaltado cómo el conocimiento limitado $\mathrm{o}$ las presunciones incorrectas sobre factores culturales y sociales al momento de desarrollar las bases para la implementación de iniciativas como las Áreas Marinas Costeras Protegidas-MU, puede impactar negativamente en los objetivos ecológicos, porque estos factores sociales y culturales serán obstáculos para la total implementación de un AMP.

A un nivel más específico, esta investigación ha tenido como objetivo analizar los elementos en la implementación de un AMP relacionados con la participación de la población local en proyectos que incluyen un enfoque de conservación y desarrollo como lo hace esta área protegida. 
La inclusión de la participación local y la consideración de elementos socio-económicos en proyectos de conservación marina han sido adoptados porque los enfoques utilizados para lograr manejar los recursos marinos habían fracasado: está siendo cada vez más claro que manejar recursos marinos tiene que ver más con conocer y entender a la gente que a los procesos ecológicos (Cinner et al. 2010). Como disciplina, la Antropología se especializa en "entender a las personas" a través de estudios sociales y culturales de la conducta humana. Sin embargo, hasta ahora, a pesar de la utilidad de la Antropología en poder investigar aspectos culturales de la conducta, ha sido principalmente reducida sólo a una categoría socio-económica de las AMP.

El enfoque Antropológico puede ir mucho más allá de estudiar elementos socio-económicos en proyectos de conservación explorando en la implementación de AMP amplificaciones sociales y culturales, por ejemplo, aquellas que se deben a la presencia o ausencia de cooperación y apoyo de los pescadores, lo cual pueden tener que ver con nociones de conceptos de 'justicia' y 'equidad' que requieran elaboración cultural (Blount y Pitchon 2007). Por ejemplo, Hall y Close (2007), exitosamente integraron conocimiento científico y local con la incorporación de antropólogos, quienes les colaboraron en la recolección de información local de pescadores sobre áreas de pesca para lograr un mejor manejo y planificación de los recursos naturales.

Aumentar la importancia de incluir a las poblaciones locales en proyectos de conservación marina (como las AMP) es necesario, porque mientras la conservación marina necesita personas que quieran participar, la decisión de participar es voluntaria.

En conservación, más aún, la gente toma opciones basadas en temas de poder y en referencia a conocimiento y accesos. Veitayaki (2002) nota como, en Fiji, la participación de las comunidades de pescadores en los proyectos de manejo de los recursos marinos los ha empoderado. Este proyecto le presenta actividades de conservación como alternativas económicas viables para las comunidades locales. Sin embargo, este tipo de participación exitosa depende de la naturaleza del involucramiento, pues este puede empoderar a algunos y al mismo tiempo desempoderar a otros (Fox et al. 2011).

Se espera que ejemplos como el ilustrado en este artículo, colaboren en promocionar el empoderamiento, participación en la toma de decisiones, inclusión y beneficios de todas las comunidades locales en los proyectos de conservación que consistan en establecer áreas protegidas. 


\section{Bibliografía}

Agrawal, A. 2005. "Environmentality, Technologies of Government and the Making of Subjects". Duke University Press, Durham and London.

Agrawal, A. y Gupta, K. 2005. "Decentralization and participation: The governance of common pool resources in Nepal's Terai". World Development 33(7), 1101-1114.

Agrawal, A. y Gibson C.C. 1999. "Enchantment and Disenchantment: The Role of Community in Natural Resource Conservation." World Development 27 (4).

Banks, S.A. y Skilleter, G.A. 2010. "Implementing marine reserve networks: A comparison of approaches in New South Wales (Australia) and New Zealand". Marine Policy 34(2), 197-207.

Bennett, N.J. y Dearden, P. 2014. "Why local people do not support conservation: community perceptions of marine protected area livelihood impacts, governance and management in Thailand". Marine Policy 44:107-116.

Bateson, G. 1979. "Espíritu y naturaleza: una unidad necesaria (avances en teoría de sistemas, complejidad y ciencias humanas)". Bantam Books.

Blomley, N. 2015. "The territory of property". Prog. Hum. Geogr. 40, 593-609. doi: 10.1177/0309132515596380

Brosius, J. P., Lowenhaupt, A. y Zerner. T. y C.1998. "Representing Communities: Histories and Politics of Communitybased Natural Resource Management". Society \& Natural Resources 11 (2): 157-68. doi: 10.1080/08941929809381069.

Bennett, N., Roth, R., Sarah C. Klain, Kai M. A. Chan, Douglas A. Clark, Cullman, G., Epstein, G., Nelson, M.P., Stedman, R., Teel, T.L. Thomas, R., Wyborn, C., Curran, D., Greenberg, A., Sandlos, J. y Verıssimo D. 2016. Mainstreaming the social sciences in conservation. Conservation Biology, Volume 00, No. 0, 1-11

Berkes, F. 2004. "Rethinking Community-Based Conservation." Conservation Biology 18 (3): 621-30.

Blount, B.G. y Pitchon, A. 2007. "An anthropological research protocol for marine protected areas: Creating a niche in a multidisciplinary cultural hierarchy". Human Organization 66(2) $103-111$.

Brooks, K. 2010. "Sustainable development: Social outcomes of structural adjustments in a South Australian fishery". Marine Policy 34(3), 671-678.

Carrier, J.G. y West, P. 2011. "Introduction: Surroundings, selves and the others: The political economy of environment and identity". Landscape Research 34(2), 157-170.

Cinner, J.E., McClanahan, T.R., y Wamukota, A. 2010. "Differences in livelihoods, socioeconomic characteristics, and knowledge about the sea between fishers and non-fishers living near and far from marine parks on the Kenyan coast". Marine Policy 34(1): 22-28. Watch.

Chapin, M. 2004. "A challenge in conservationists". World

Chuenpagdee, R., J. Pascual-Fernández, J. Szeliánszky, E. Alegret J.L. Fraga, J. y Jentoft. S. 2013. "Marine Protected
Areas: Re-Thinking Their Inception." Marine Policy 39 (1): 234-40. doi:10.1016/j.marpol.2012.10.016.

Curtin, R. \& Prellezo, R. 2010. Understanding marine ecosystem based management: A literature review. Marine Policy 34(5), 821-830.

Diegues A.C.; Chapin, M. (Org.) ; Ottaway D. (Org.) ; Stephens J. (Org.) ; Compagnon D. (Org.) ; Diaw, M.C (Org.) ; Dowie M. (Org.) ; Brockington, D. (Org.) ; Igoe, J. (Org.) . 2008. "A ecologia política das grandes ONGs transnacionais conservacionistas". 1. ed. São Paulo: Nupaub/USP, v. 1.198p

Deguignet, M., Juffe-Bignoli, D., Harrison, J., MacSharry, B., Burgess, N. y Kingston, N. 2014. "United Nations List of Protected Areas. United Nations Environment Programme". UNEP-WCMC: Cambridge. UK.

Durand, L. y Vázquez, L. B. 2011. "Biodiversity conservation discourses. A case study on scientists and government authorities in Sierra de Huautla Biosphere Reserve, Mexico". Land Use Policy 28(1), 76-82.

Evans-Pritchard, E.E. 1940. "The Nuer". Clarendon Press. Oxford.

Fox, H.E., Mascia, M. B., Basurto, X., Costa, A., Glew, L., Heinemann, D., Karrer, L.B., Lester, S.E., Lombana, A.V., Pomeroy, R.S., Recchia, Ch.A., Roberts, C.M., Sanchirico, J.N., Pet-Soede, L. y White, A.T. 2011. "Reexamining the science of marine protected areas: linking knowledge to action". Conservation Letters 5, 1-10.

Gelcich, S., Edwards-Jones, G. y Kaiser, M. J. 2005. "Importance of attitudinal differences among artisanal fishers toward co-management and conservation of marine resources". Conservation Biology, 19(3):865-875.

Gerhardinger, L., Gorris P., Leandra R. Gonçalves, L., Herbst, D., Vila-Nova, D., De Carvalho, F., Glaser, M., Zondervan, R. y Glavovic, B. 2018. "Healing Brazil's Blue Amazon: The Role of Knowledge Networks in Nurturing Cross-Scale Transformations at the Frontlines of Ocean Sustainability". Frontiers in Marine Science. ORIGINAL RESEARCH published: 26 January 2018 doi: 10.3389/fmars.2017.00395

Guerreiro, J., Chircop, A., Grilo, C., Viras, A., Ribeiro, R. y van der Elst, R. 2010. "Establishing a transboundary network of marine protected areas: Diplomatic and management options for the east African context". Marine Policy 34(5), 896-910.

Guha, R. 2000. "The paradox of global environmentalism". Current History, 99(640), 367-37

Hall, G.B. \& Close C.H. 2007. Local knowledge assessment for a small-scale fishery using geographic information systems. Fisheries Research 83, 11-22.

Halpern, B.S., Walbridge, S. Selkoe, K.A., Kapel, C.V., Micheli F., D'Agrosa, C., Bruno, J.F., Casey K.S., Ebert, C., Fox, H.E., Fujita R., Heinemann, D., Lenihan, H.S., Madin, E.M., Perry, M.T., Selig, E.R., Spalding, M., Steneck, R., Watson, R. 2008. "A global map of human impact on marine ecosystems". Science. 319 (5865): 
$948-52$.

Herrold-Menzies, M. 2006. "Integrating conservation and development: What we can learn from Caohai, China". Journal of Environment and Development 15(4), 382-406.

Hughes, R., y Flintan, F. 2001. "Integrating Conservation and Development Experience: Biodiversity and Livelihoods Issues". No. 3. IIED, London, UK.

Hutton, J., Adams, W.M. y Murombedzi J.C. 2005. "Back to the barriers? Changing narratives in biodiversity conservation". Forum for Development Studies 32(2), 341-370.

Koehn, J. Z., Reineman, D. R., and Kittinger, J. N. 2013. "Progress and promise in spatial human dimensions research for ecosystem-based ocean planning". Mar. Policy 42, 31-38. doi: 10.1016/j.marpol.2013.01.015

Kopnina, H. y Shoreman-Ouimet, E. 2013. "Environmental anthropology: future directions". Edited by Helen and Routledge, London, Jul 18, 2013 - 314 pages.

Kelleher, G. y Recchia, C. 1998. "Lessons from Marine Protected Areas around the world". Parks 8: 1-4.

Lockyer J. y Veteto, J. R. 2013 "Environmental Anthropology Engaging Ecotopia, Bioregionalism, Permaculture, and Ecovillages". Published by: Berghahn Books.

Ma, C., Zhang, X., Chen, W., Zhang, G., Duan, H. Ju, M. Li, H. y Yang, Z. 2013. "China's special marine protected area policy: Trade-off between economic development and marine conservation". Ocean and Coastal Management (76): 1-11.

Mascia, M.B., Claus, C.A. \& Naidoo, R. 2010. Impacts of marine protected areas on fishing communities. Conservation Biology 24(5), 1424-1429.

Nepal, S.K. 2002. "Involving indigenous peoples in protected area management: Comparative perspectives from Nepal, Thailand, and China". Environmental Management 30(6), 748-763.

Netting, R. McC. 1968. "Hill Farmers of Nigeria; Cultural Ecology of the Kofyar of the Jos Plateau". University of Washington Press. Seattle, USA.

Netting, R. McC. 1964 "Household organization and intensive agriculture: The Kofyar case". Africa 35, 422-29.

Netting, R. McC. 1972. "Of men and meadows: Strategies of Alpine land use". Anthropology 45, 132-44.0.

Milton, K. (Ed.). 1993. "Environmentalism the view from Anthropology". London: Routledge.

Pinkerton, E. y John, L. 2008. "Creating local management legitimacy”. Marine Policy, 32(4): 680-691.
Rappaport, R.A. 1968. "Pigs for the Ancestors: Ritual in the Ecology of a New Guinea People". Yale University Press. New Haven, CT, USA.

Ross, H., Grant, C., Robinson, C.J., Izurieta, A., Smyth, D. y Rist, P. 2009. "Co-management and Indigenous protected areas in Australia: Achievements and ways forward". Australasian Journal of Environmental Management 16(4), 242-252.

Kenneth, R., Francis R., Hickey, F. y Hickey. F.R. 2008. "Accounting for the Mismanagement of Tropical Nearshore Fisheries." Environment, Development and Sustainability 10 (5): 565-89. doi: 10.1007/s10668-008-9152-5.

Sanchirico, J.N., Cochran, K.A. y Emerson, P.M. 2002. "Marine Protected Areas: Economic and Social Implications. Resources for the Future, Discussion" Paper 02e26. http:// www.cbd.int/doc/ case-studies/inc/cs-inc-rf-04-en.pdf (accessed 3/2011).

Shoreman-Ouimet, E. y Kopnina H. 2016. "Culture and Conservation: Beyond Anthropocentrism". Routledge, Oxfordshire, UK.

Sievanen, L. 2009. "Environmental Anthropology". Anthropology 5400. Course description at University of North Texas. USA.

Schmidt, P.M. \& Peterson, M.J. 2009. Biodiversity conservation and indigenous land management in the era of self-determination. Conservation Biology 23(6), 1458-1466.

Townsend, P.K. 2000. "Environmental Anthropology: From Pigs to Policies. Prospect Heights". IL: Waveland Press, Inc.

Veitayaki, J. 2002. "Customary marine tenure and empowerment resource owners in Fiji". In: South, G.R., Cleave, G. and Skelton, P.A. (eds.), Oceans in the New Millennium: Challenges and Opportunities for the Islands. Proceedings of the Pacem in Maribus XXVII, Bucharest, Dada. Pp. 148-150.

Wapner, P. y Matthew R.A. 2009. "The humanity of global environmental ethics". The Journal of Environment \& Development 18(2), 203-222.

West, P. 2006. "Conservation Is Our Government Now". Durham and London Duke $U$

West, P. 2012 "From Modern Production to Imagined Primitive: The Social World of Coffee from Papua New Guinea". Published: University Press.

WWF 2007. Strengthening Governance and Sustainable Livelihoods in the Huilliche Territory of Mapu Lahual Valdivia: Proposal to the New Zealand Agency for International Development (Report). 